\title{
Multiscale Geometry and Scaling of the Turbulent-Nonturbulent Interface in High Reynolds Number Boundary Layers
}

\author{
Charitha M. de Silva, Jimmy Philip, Kapil Chauhan, Charles Meneveau,* and Ivan Marusic \\ Department of Mechanical Engineering, University of Melbourne, Victoria 3010, Australia \\ (Received 5 March 2013; revised manuscript received 4 June 2013; published 24 July 2013)
}

\begin{abstract}
The scaling and surface area properties of the wrinkled surface separating turbulent from nonturbulent regions in open shear flows are important to our understanding of entrainment mechanisms at the boundaries of turbulent flows. Particle image velocimetry data from high Reynolds number turbulent boundary layers covering three decades in scale are used to resolve the turbulent-nonturbulent interface experimentally and, for the first time, determine unambiguously whether such surfaces exhibit fractal scaling. Box counting of the interface intersection with the measurement plane exhibits power-law scaling, with an exponent between -1.3 and -1.4 . A complementary analysis based on spatial filtering of the velocity fields also shows power-law behavior of the coarse-grained interface length as a function of filter width, with an exponent between -0.3 and -0.4 . These results establish that the interface is fractallike with a multiscale geometry and fractal dimension of $D_{f} \approx 2.3-2.4$.
\end{abstract}

DOI: 10.1103/PhysRevLett.111.044501

The geometry of turbulence, with visually striking eddy motions at many scales, has fascinated observers as far back as Leonardo da Vinci [1]. Particularly striking are surfaces in turbulent flows that are made visible through various means. Examples include cumulus cloud boundaries [2], turbulent flames [3], and the visibly sharp and corrugated surface separating turbulence from outer, nonturbulent regions in shear flows such as jets and boundary layers [4-7]. While each of these surfaces are affected by various different physical processes, a common effect is the uneven advection and surface deformation due to turbulent eddies that tend to increase the surface area. Also common are molecular processes that typically occur at small diffusive scales, which tend to smooth, i.e., reduce, the surface area. Early work in the 1980s and 1990s emphasized the multiscale structure of interfaces as a reflection of deformations caused by a hierarchy of eddy sizes in the turbulent inertial range. Following proposals that surfaces may display scale-invariant (fractal) properties [8], experimental results were reported with evidence of such surfaces having a fractal dimension of $D_{f} \approx 2.3-2.4$. Early and more recent observations consistent with fractal scaling included cloud boundaries [2,9], turbulent flames in the corrugated flamelet regime $[3,10,11]$, and interfaces determined from isoconcentration surfaces of passive scalars [12]. Moreover, scaling arguments were provided connecting the fractal dimension to the classical Kolmogorov scaling of turbulence. One considers [13] an interface separating two regions across which a transported quantity $C$ exhibits a finite concentration difference $\Delta C$, and one evaluates the flux across the surface using descriptions at various coarse-graining lengths $r$. The total flux is the product of the effective diffusion coefficient, the gradient, and the total surface area at that scale, $S(r)$. The gradient scales as $\Delta C / r$
PACS numbers: 47.53.+n, 02.50.Fz, 47.27.N-, 47.27.T-

and the turbulent effective diffusivity scales as $\epsilon^{1 / 3} r^{4 / 3}$, if $r$ belongs to the inertial range and $\epsilon$ is the rate of dissipation (Kolmogorov scaling, i.e., Richardson relative diffusivity, is assumed). Thus, the total flux scales as $\left\{\epsilon^{1 / 3} r^{4 / 3-1} S(r) \Delta C\right\}$. For it to be independent of $r$ (i.e., if the turbulent diffusion correctly reproduces the unresolved mixing at scales below $r$ ), the surface area must follow $S(r) \sim r^{-1 / 3}$. Power-law scaling of the surface area is a hallmark of fractals, with $S(r) \sim r^{2-D_{f}}$. Hence, $D_{f}=2+$ $(1 / 3)$, where the $1 / 3$ term originates from Kolmogorov scaling. Arguments based on the molecular fluxes evaluated at the diffusive scales and then invoking Reynolds number similarity lead to the same result [12].

There has been renewed interest in the structure of turbulence, entrainment, and transport in the vicinity of the turbulent-nonturbulent interface (TNTI) (see e.g., [7,14-18], and [19,20]). Recent studies on TNTI have focused on the question of whether the growth rate of the turbulent region into the nonturbulent region is determined by large-scale processes through big eddies (engulfment), or by small-scale diffusive processes occurring at the interface (viscous nibbling). Evidence for both viewpoints has been presented. While the question has not been settled thus far, as suggested by Ref. [14], the arguments invoking a multiscale fractal structure of the interface $[12,13]$ can be used to reconcile the two views: the engulfment due to inertial eddy structures of large and many intermediate sizes is responsible for an increased surface area, along which diffusive processes can act more extensively.

And yet, while the above argument appears reasonable and consistent with traditional expectations, the direct evidence from prior experiments and simulations for fractal scaling of interfaces is inconclusive. There are considerable practical difficulties associated with providing conclusive evidence for or against fractal (power-law) 
scaling. Lack of convincing power-law scaling (e.g., curving in log-log plots) was quoted in various prior works, see, e.g., [21-25]. Most of these previous studies used passive scalars as surrogate markers to identify the interface, with concomitant uncertainties of interpretation, and were in flows with only moderately high Reynolds numbers, where a limited range of length scales exist, thus, restricting the length or possibly the emergence of a well-established power-law scaling. As a result, the notion of power-law scaling of interfaces in turbulence is not yet generally accepted in the literature. Ideally, very high Reynolds numbers are required in order to isolate power-law scaling over a subrange of scales between the inner cutoff scale (e.g., the Kolmogorov scale $\eta$ ) and the outer (integral) scale, $\delta$. Here we overcome the concomitant experimental challenges by employing one of the largest zero-pressure gradient wind tunnels and multiple high-resolution sensors to acquire data.

High resolution particle image velocimetry (PIV) proves to be an effective technique to study the multiscale dynamics of the interface. For example, PIV has been used in jets at $\operatorname{Re}_{D}=2 \times 10^{3}$ to measure viscous enstrophy fluxes near the TNTI [15], as well as the influence of shear and swirl on the TNTI [16]. In this Letter, we reexamine the fractal aspects of the TNTI using two experimental data sets in very high Reynolds number turbulent boundary layers, the parameters of which are summarized in Fig. 1.

In order to resolve the full range of scales, eight high resolution cameras (each with $4008 \times 2672$ pixels) were used simultaneously. A merged instantaneous image across the eight cameras provides a field of view of $\approx 0.73 \mathrm{~m} \times$ $0.4 \mathrm{~m}$ in the streamwise $(x)$ and wall-normal $(y)$ directions, respectively, corresponding to $1538 \times 839$ vectors in $x$ and $y$. The images are processed with a vector spacing of $0.64 \eta^{a}$ and $1.21 \eta^{b}$, or 10.3 and 19.4 wall units $\left(\nu / u_{\tau}\right)$,

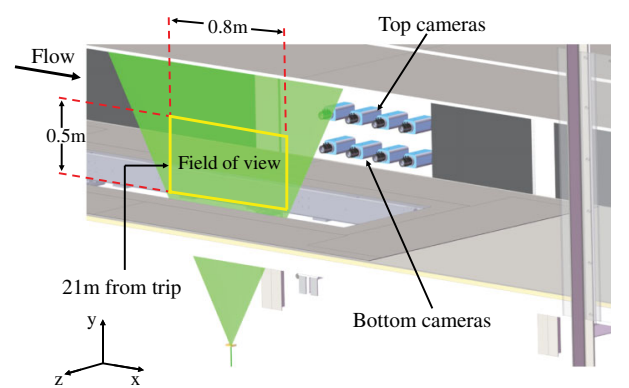

$\begin{array}{llllll}\operatorname{Re}_{\tau} & \operatorname{Re}_{\delta} \times 10^{-5} & U_{\infty} & u_{\tau}=\sqrt{\tau_{w} / \rho} \quad \delta \quad \eta \times 10^{4} & \lambda_{T} \times 10^{3}\end{array}$

\begin{tabular}{ccccccc}
$\left(u_{\tau} \delta / \nu\right)$ & $\left(U_{\infty} \delta / \nu\right)$ & {$\left[\mathrm{ms}^{-1}\right]$} & {$\left[\mathrm{ms}^{-1}\right]$} & {$[\mathrm{m}]$} & {$[\mathrm{m}]$} & {$[\mathrm{m}]$} \\
\hline $7900^{a}$ & 2.37 & 10.08 & 0.334 & 0.363 & 7.4 & 10.1 \\
$14500^{b}$ & 4.6 & 20.04 & 0.630 & 0.353 & 3.9 & 7.3
\end{tabular}

FIG. 1 (color online). Experimental setup for the planar PIV measurements in the High Reynolds Number Boundary Layer Wind Tunnel at the University of Melbourne, and table of relevant flow properties, where $\eta$ and $\lambda_{T}$ are the Kolmogorov and Taylor microscales calculated near the interface and $\tau_{w}$ is the wall stress [26]. for $\operatorname{Re}_{\tau}=7900$ and 14500 , respectively. For each Reynolds number, 1000 vector fields are analyzed to provide converged first and second order statistics. Further details on the experimental setup, PIV processing details, and the validation of the planar PIV data against prior hotwire anemometry measurements can be found in [26]. These data are well suited for the present analysis: they have a large field of view $(\approx 2 \delta$ in $x$ and $1.1 \delta$ in $y$ ), and consist of $\mathcal{O}\left(10^{3}\right)$ vectors in each direction, resolving from scales close to the Kolmogorov scale up to scales on the order of $\delta$ in a high Reynolds number flow, providing an available scale range of $2 \delta / \eta \approx 10^{3}$. Such a measurement based directly on velocity fields and a large scale range enables us to ascertain conclusively if any power-law scaling exists.

Various approaches have been proposed to detect the TNTI from velocity field or visualization results, some based on vorticity $[27,28]$ or passive scalar markers $[7,12]$. For our data, we have found that isosurfaces of the local (and two component) kinetic energy $K=(1 / 2) \times$ $\left(u^{2}+v^{2}\right)$ of the flow in the frame moving with the free stream provides a very good indicator of the interface. Compared to vorticity-based methods that depend on derivatives, the kinetic energy is less susceptible to noise and finite resolution. While the kinetic energy field is dominated by the large-scale eddies, its isosurfaces contain geometric fluctuations down to the Kolmogorov scale. Figure 2 shows a sample PIV image and the interface determined based on the threshold of $K=c_{0}\left((1 / 2) U_{\infty}^{2}\right)$ with $c_{0}=1 \times 10^{-3}$ (thresholds of $c_{0}=0.5 \times 10^{-3}$ and $2 \times 10^{-3}$ are also tested). In order to exclude small islands that could be due to low-intensity pockets inside the turbulent region arising from "inner intermittency", or from

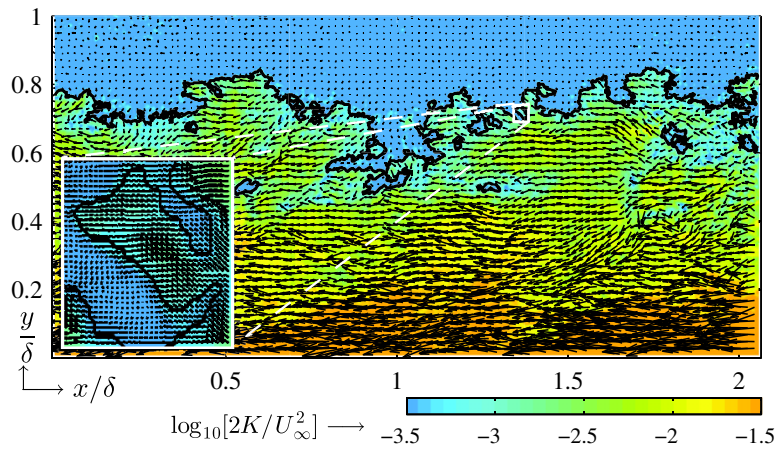

FIG. 2 (color online). A snapshot of the velocity field relative to the free stream at $\operatorname{Re}_{\tau}=14500$ from planar PIV. Every 10th vector is plotted in each direction. Color contours represent kinetic energy $K=(1 / 2)\left(u^{2}+v^{2}\right)$, where velocities are in a frame moving with the free stream. The solid black line indicates the location of the TNTI computed as an isokinetic energy surface using a threshold of $K=10^{-3}\left((1 / 2) U_{\infty}^{2}\right)$. The inset shows a magnified view of the TNTI, showing vectors at the original resolution, and from $1.34<x / \delta<1.39$ and $0.69<$ $y / \delta<0.74$. A velocity magnitude of $8 \mathrm{~m} / \mathrm{s}$ corresponds to a length of $0.1 \delta$ in the main vector map, and $0.03 \delta$ in the inset. 
experimental noise in the outside region above the interface, only islands with an area greater than $\lambda_{T}^{2}$ are counted towards the TNTI, where $\lambda_{T}$ is the Taylor microscale. As shown in Fig. 2, the results from this detection method agree well with the interface location as it can be identified visually in a more qualitative fashion. While the appropriate threshold to be used may depend on the flow, the level of free-stream (background) turbulence, signal noise $\left(\approx 0.2 \%\right.$ and $1 \%$ of $U_{\infty}$, respectively), etc., quantitatively robust results with respect to specific choices of the threshold are obtained.

In order to determine whether the data exhibit fractal scaling, a box-counting analysis is performed by counting $N(r)$, the number of boxes of size $r$ required to cover the TNTI, for a wide range of box sizes. For fractal scaling with dimension $D$, one expects $N(r) \sim r^{-D}$. In the analysis, $N(r)$ is averaged over the 1000 individual fields, and divided by the length of the projected interface in the $x$ direction $\left(L_{x}\right)$. The resulting number of boxes per unit length are plotted in Fig. 3 in log-log axes for both data sets at the two Reynolds numbers. The inset shows the "local slope" obtained by evaluating the logarithmic derivative using centered finite difference of consecutive data points. Power-law scaling is clearly exhibited from a large-scale cutoff near $\approx 20 \%$ of $\delta$ down to small scales where the viscous cutoff scale, or spatial resolution of the data are expected to begin to smooth the results. Vertical lines are drawn at $10 \eta$ where no smoothing effects of spatial resolution are expected, i.e., a conservative lower limit for determining the power-law exponent, although it is apparent that fractal scaling may extend to even smaller scales. The average slope is near -1.3 , consistent with a fractal dimension of the perimeter $D \approx 1.3$, and with $D_{f} \approx 2.3$

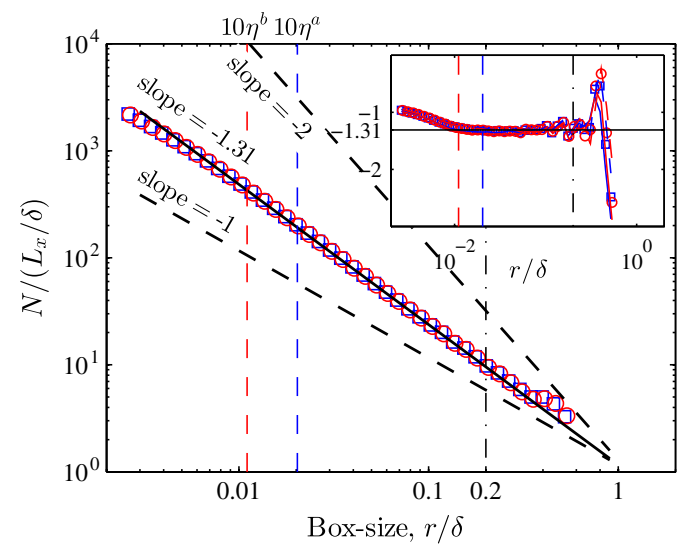

FIG. 3 (color online). Plot of the number of boxes $N(r)$ needed to cover the TNTI at a kinetic energy threshold of $0.1 \%$. Squares: $\operatorname{Re}_{\tau}=7900$ and circles: $\operatorname{Re}_{\tau}=14500$. For comparison, dashed lines show slopes of -1 and -2 . Vertical lines show the limits used for the fit for each set of data. The inset shows the local slopes; the solid and dashed lines $\left(c_{0}=0.05 \%\right.$ and $\left.0.2 \%\right)$, and symbols $(0.1 \%)$ which correspond to the TNTI detected using different thresholds, where, $K=c_{0}\left((1 / 2) U_{\infty}^{2}\right)$. for the surface embedded in three dimensions according to the additive rule of co-dimensions for intersecting sets [8]. At the smaller scales the slope tends to one, consistent with the expectation that fractal scaling ceases below an inner cutoff or when approaching the spatial resolution of the data. In terms of sensitivity to thresholding, the inset in Fig. 3 shows no significant variation in the local slope when using different thresholds $\left(c_{0}=0.05 \%, 0.1 \%\right.$, and $0.2 \%$ ); furthermore, a reduced convergence above $0.1 \delta$, due to the reduced number of samples obtained at large $r$ is also observed.

An understanding of the multiscale properties of interfaces is particularly useful in the context of describing the physics at various length scales. Coarse graining is commonly used in large eddy simulations (LES) of turbulence [29], in which the velocity field is spatially filtered with a smoothing kernel of characteristic scale $\Delta$, according to $\tilde{u}_{i}(\mathbf{x}, t)=\int u_{i}\left(\mathbf{x}-\mathbf{x}^{\prime}, t\right) G_{\Delta}\left(\mathbf{x}^{\prime}\right) d^{3} \mathbf{x}^{\prime}$. Here, $\tilde{u}_{i}(\mathbf{x}, t)$ is the filtered velocity that would be simulated in LES. Experimental data from PIV can be used to study scaleto-scale interactions in turbulence in physical space by filtering the velocity at various scales $\Delta[30]$. Here, we examine the scale-to-scale properties of the TNTI by coarse graining the velocity using a $2 \mathrm{D}$ box filter on the PIV data (i.e., $G_{\Delta}(x, y)=1 / \Delta^{2}$ if $|x|,|y|<\Delta / 2$ and $G_{\Delta}(x, y)=0$ otherwise). Then the kinetic energy of the coarse-grained velocity field is evaluated according to $K^{\Delta}=(1 / 2)\left(\tilde{u}^{2}+\tilde{v}^{2}\right)$ and the same threshold $(0.1 \%$ of $(1 / 2) U_{\infty}^{2}$, the free-stream kinetic energy in the wallattached frame) is used to define the interface. As before, islands whose area is smaller than $\lambda_{T}^{2}$ are excluded from the TNTI. As shown in Fig. 4, when $\Delta$ is small, on the order of the distance between PIV vectors, the interface is the same as that shown in Fig. 2. For larger $\Delta$ (but with the same threshold), the interface becomes progressively smoother. The interface location shows no bias shift in position when computed from the filtered velocity fields as shown in

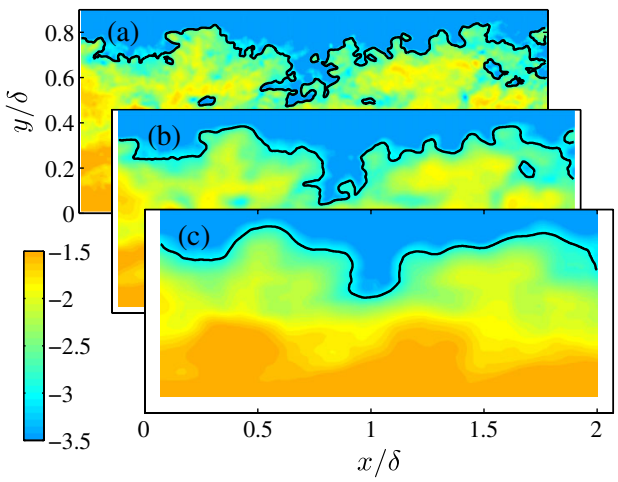

FIG. 4 (color online). TNTI determined from the filtered velocity fields at a kinetic energy threshold of $0.1 \%$, with filter scales (a) $\Delta=0.02 \delta$, (b) $\Delta=0.08 \delta$, and (c) $\Delta=0.18 \delta$. Color contours represent the filtered kinetic energy, $K^{\Delta}=$ $(1 / 2)\left(\tilde{u}^{2}+\tilde{v}^{2}\right)$ in logarithmic units, in a frame moving with the free stream. 

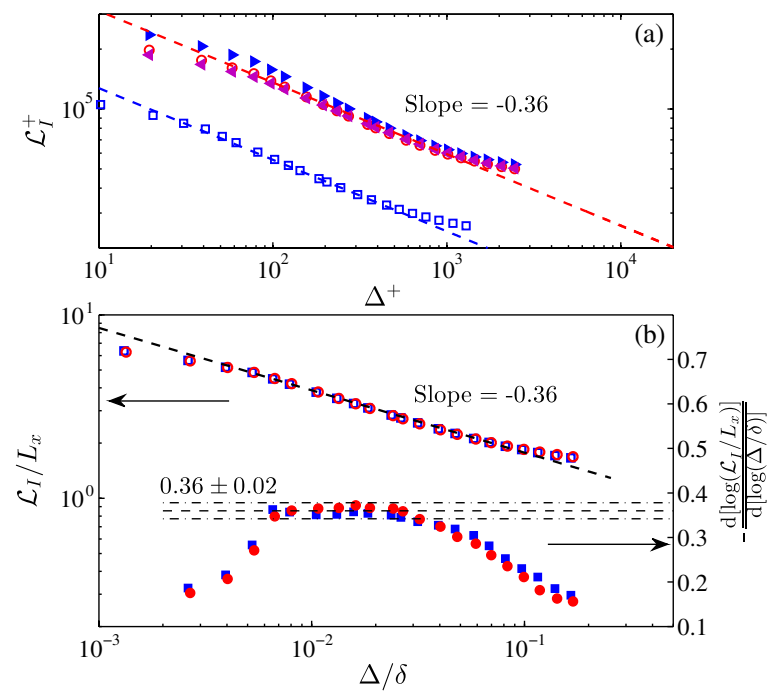

FIG. 5 (color online). Average measured interface length as function of filter scale. (a) Normalized with viscous units for data at $\operatorname{Re}_{\tau} \approx 7900$ (open squares) and $\operatorname{Re}_{\tau} \approx 14500$ (open circles). At $\operatorname{Re}_{\tau} \approx 14500$, three thresholds are considered, namely $0.05 \%, 0.1 \%$, and $0.2 \%$ of $(1 / 2) U_{\infty}^{2}$ and indicated by right triangles, open circles, and left triangles, respectively. (b) Average measured interface length normalized by the length of the PIV image $\left(L_{x}\right)$ and $\delta$, for a $0.1 \%$ kinetic energy threshold. Note that $L_{x}$ decreases with increasing $\Delta$ as a larger region on the edges has to be excluded. Closed squares and closed circles: local slope computed using central differences. The slope uncertainty of \pm 0.02 in Fig. 5(b) is determined by the maximum deviation of the slope in the range $6 \times 10^{-3}<\Delta / \delta<3 \times 10^{-2}$ for both data sets.

Fig. 4, indicating that the TNTI criterion based on kinetic energy is suitable for velocity fields at various scales, such as those from LES.

Of particular interest is the surface area as a function of scale. From the planar PIV data, the corresponding quantity we can measure is the length $\mathcal{L}_{I}$ of the TNTI as a function of filter scale $\Delta$, and for a fractal boundary one expects $\mathcal{L}_{I} \sim \Delta^{1-D}$. The results are shown in Fig. 5(a) for the two Reynolds number data sets and for three thresholds $\left(c_{0}=0.05 \%, 0.1 \%\right.$, and $\left.0.2 \%\right)$ for the high Reynolds number case. Length and filter sizes are normalized on viscous units, i.e., $\mathcal{L}_{I}^{+}=\mathcal{L}_{I} u_{\tau} / \nu$ and $\Delta^{+}=\Delta u_{\tau} / \nu$. The dashed lines show fits in the scaling region between $50<\Delta^{+}<$ 300 and $100<\Delta^{+}<600$ at $\operatorname{Re}_{\tau} \approx 7900$ and 14500 , respectively. The threshold of $0.05 \%$ yields a slightly steeper slope $(\approx-0.4)$, although at this threshold the detected interface already appears more noisy and corresponds less visibly to the TNTI. Figure 5(b) shows the same results (only $c_{0}=0.1 \%$ to avoid clutter), displaying collapse when plotted in $\Delta / \delta$. A plateau is evident in the logarithmic derivative of the measured length of TNTI (solid symbols), with a mean of $\approx 0.35$, consistent again with $D \approx 1.3-1.4$ or $D_{f} \approx 2.3-2.4$. As a caveat, due to $x-y$ plane data, flow anisotropy effects cannot be excluded, especially at large scales. At inertial range and small scales, however, the expectation of isotropy in interface corrugation suggests our results are independent of measurement plane orientation.

While the prior published evidence for power-law scaling of the TNTI was inconclusive and the applicability of fractals to describe interfaces not established, the results introduced here provide compelling evidence that the TNTI in turbulent boundary layers (an open shear flow) display fractal-like power-law scaling, clearly consistent with $D_{f} \approx 2.3$ to 2.4 . Future work will focus on implications of the present geometric analysis on entrainment fluxes as function of scale.

The authors thank the Australian Research Council for the financial support. C.M.'s visit to the University of Melbourne was supported by the Australian-American Fulbright Commission Senior Scholar Fellowship and a University of Melbourne MERIT Visiting Scholarship.

*Permanent address: Department of Mechanical Engineering and Center for Environmental and Applied Fluid Mechanics, The Johns Hopkins University, 3400 North Charles Street, Baltimore, Maryland 21218, USA.

[1] M. Gad-El-Hak, Passive, Active and Reactive Flow Management (Cambridge University Press, Cambridge, England, 2000).

[2] S. Lovejoy, Science 216, 185 (1982).

[3] F. Gouldin, K. Bray, and J. Chen, Combust. Flame 77, 241 (1989).

[4] S. Corrsin and A. L. Kistler, NACA Tech. notes, 1244 (1955).

[5] O. M. Phillips, Math. Proc. Cambridge Philos. Soc. 51, 220 (1955).

[6] K. Sreenivasan and C. Meneveau, J. Fluid Mech. 173, 357 (1986).

[7] J. Westerweel, C. Fukushima, J. M. Pedersen, and J. C. R. Hunt, J. Fluid Mech. 631, 199 (2009).

[8] B. B. Mandelbrot, The Fractal Geometry of Nature (W. H. Freeman, New York, 1982).

[9] A. P. Siebesma and H. J. J. Jonker, Phys. Rev. Lett. 85, 214 (2000).

[10] R. Knikker, D. Veynante, and C. Meneveau, Phys. Fluids 16, L91 (2004).

[11] E. R. Hawkes, O. Chatakonda, H. Kolla, A. R. Kerstein, and J. H. Chen, Combust. Flame 159, 2690 (2012).

[12] K. Sreenivasan, R. Ramshankar, and C. Meneveau, Proc. R. Soc. A 421, 79 (1989).

[13] C. Meneveau and K. R. Sreenivasan, Phys. Rev. A 41, 2246 (1990).

[14] J. Mathew and A. Basu, Phys. Fluids 14, 2065 (2002).

[15] J. Westerweel, C. Fukushima, J. M. Pedersen, and J. C. R. Hunt, Phys. Rev. Lett. 95, 174501 (2005).

[16] J. C. R. Hunt, I. Eames, C. B. da Silva, and J. Westerweel, Phil. Trans. R. Soc. A 369, 811 (2011).

[17] J. Philip and I. Marusic, Phys. Fluids 24, 055108 (2012).

[18] M. Holzner and B. Lüthi, Phys. Rev. Lett. 106, 134503 (2011). 
[19] J. Katzwinkel, H. Siebert, and R. A. Shaw, Bound.-Lay. Meteorol. 145, 131 (2012).

[20] D. M. Romps, J. Atmos. Sci. 67, 1908 (2010).

[21] P. L. Miller and P.E. Dimotakis, Phys. Fluids A 3, 168 (1991).

[22] H. J. Catrakis and C.L. Bond, Phys. Fluids 12, 2295 (2000).

[23] R.D. Frederiksen, W. J.A. Dahm, and D. Dowling, J. Fluid Mech. 338, 89 (1997).

[24] H. J. Catrakis, R. Aguirre, and J. Ruiz-Plancarte, J. Fluid Mech. 462, 245 (2002).

[25] H. J. Catrakis, R.C. Aguirre, J. Ruiz-Plancarte, R. D. Thayne, B. A. McDonald, and J.W. Hearn, J. Fluid Mech. 471, 381 (2002).
[26] C.M. de Silva, K.A. Chauhan, C.H. Atkinson, N. A. Buchmann, N. Hutchins, J. Soria, and I. Marusicin, in 18th Australasian Fluid Mechanics Conference, Australia, 2012, edited by P.A. Brandner and B. W. Pearce (Australasian Fluid Mechanics Society, Melbourne, Australia, 2012).

[27] D. Bisset, J. C. R. Hunt, and M. M. Rogers, J. Fluid Mech. 451, 383 (2002).

[28] J. Jimenez, S. Hoyas, M.P. Simens, and Y. Mizuno, J. Fluid Mech. 657, 335 (2010).

[29] C. Meneveau and J. Katz, Annu. Rev. Fluid Mech. 32, 1 (2000).

[30] J. Chen, C. Meneveau, and J. Katz, J. Fluid Mech. 562, 123 (2006). 